\title{
Nutrient profiling and adherence to components of the UK national dietary guidelines association with metabolic risk factors for CVD and diabetes: Airwave Health Monitoring Study
}

\author{
Rebeca Eriksen ${ }^{1}$, Rachel Gibson ${ }^{1}$, Kathryn Lamb ${ }^{1}$, Yvonne McMeel $^{1}$, Anne-Claire Vergnaud ${ }^{2}$, \\ Jeanette Spear ${ }^{2}$, Maria Aresu ${ }^{2}$, Queenie Chan $^{2}$, Paul Elliott ${ }^{2}$ and Gary Frost ${ }^{1 *}$ \\ ${ }^{1}$ Nutrition and Dietetic Research Group, Imperial College, Faculty of Medicine, London W12 ONN, UK \\ ${ }^{2}$ Department of Epidemiology and Biostatistics, School of Public Health, MRC-PHE Centre for Environment and Health, \\ Imperial College London, London W2 1PG, UK
}

(Submitted 26 April 2017 - Final revision received 7 December 2017 - Accepted 12 December 2017-First published online 19 February 2018)

\section{Abstract}

CVD is the leading cause of death worldwide. Diet is a key modifiable component in the development of CVD. No official UK diet quality index exists for use in UK nutritional epidemiological studies. The aims of this study are to: (i) develop a diet quality index based on components of UK dietary reference values (DRV) and (ii) determine the association between the index, the existing UK nutrient profile (NP) model and a comprehensive range of cardiometabolic risk markers among a British adult population. A cross-sectional analysis was conducted using data from the Airwave Health Monitoring Study ( $n$ 5848). Dietary intake was measured by 7 -d food diary and metabolic risk using waist circumference, BMI, blood lipid profile, glycated $\mathrm{Hb}$ (HbA1c) and blood pressure measurements. Diet quality was assessed using the novel DRV index and NP model. Associations between diet and cardiometabolic risk were analysed via multivariate linear models and logistic regression. A two-point increase in NP score was associated with total cholesterol $(\beta-0.33 \mathrm{mmol} / 1, P<0 \cdot 0001)$ and HbA1c $(\beta-0 \cdot 01 \%$, $P<0.0001)$. A two-point increase in DRV score was associated with waist circumference $(\beta-0.56 \mathrm{~cm}, P<0.0001), \mathrm{BMI}\left(\beta-0.15 \mathrm{~kg} / \mathrm{m}^{2}\right.$, $P<0.0001)$, total cholesterol $(\beta-0.06 \mathrm{mmol} / 1, P<0.0001)$ and HbA1c $(\beta-0.02 \%, P=0.002)$. A one-point increase in DRV score was associated with type 2 diabetes (T2D) (OR $0.94, P=0.01$ ) and obesity (OR $0.95, P<0.0001$ ). The DRV index is associated with overall diet quality and risk factors for CVD and T2D, supporting its application in nutritional epidemiological studies investigating CVD risk in a UK population.

\section{Key words: Diet quality: Nutrient profile: Type 2 diabetes: CVD}

Worldwide CVD is the number one cause of death accounting for $31 \%$ of deaths in 2012 ( 17.5 million) and predicted to be 23.4 million deaths by $2030^{(1)}$. The risk of CVD increases with the number of metabolic risk factors present including elevated blood sugar, high blood pressure (BP), dyslipidaemia and abdominal obesity ${ }^{(2)}$. A combination of three or more of these risk factors are referred to as the metabolic syndrome (MetS) ${ }^{(3)}$.

A person who has the MetS is twice as likely to develop CVD and five times as likely to develop type 2 diabetes (T2D) as someone who has less than two risk factors ${ }^{(2)}$. In the UK, almost one in four have the MetS - 20\% in men ${ }^{(4)}$ and $29 \%$ in women $^{(5)}$. In this context reducing these metabolic risk factors is of major importance in the prevention of CVD and T2D.

The MetS is multi-factorial ${ }^{(6-8)}$. Some of the driving forces causing the MetS are obesity, poor diet quality and physical inactivity ${ }^{(7)}$. Diet quality is a key modifiable component in the development of these cardiometabolic risk factors, as demonstrated in many randomised control trials and epidemiological studies $^{(8-14)}$. Studies have shown a holistic approach to dietary assessment, for example a diet quality index is a good measure to capture the combined quality and effect of nutrients in a person's diet in relation to cardiometabolic health ${ }^{(13,15-24)}$. Evidence suggests a diet quality model may need to be countryspecific to its study population ${ }^{(14,15,25,26)}$. A critical review of twenty diet quality models found that they differ in many aspects, for example dietary components included and cut-off values used ${ }^{(15)}$. This suggests that not all adequately capture dietary components related to specific population's diet and health outcomes ${ }^{(14,15)}$. The main argument is that the models are often derived from a specific population and may not be suitable in capturing important foods consumed in other population groups ${ }^{(14,15,25,26)}$.

No official UK diet quality index exists to measure overall diet quality in nutritional epidemiological studies of the UK population. One potential method is to apply the UK nutrient profile (NP) model score developed by the Food Standards Agency in 2005. The NP model assesses quality of individual foods based on the national dietary guidelines and the Eatwell Guide ${ }^{(27-29)}$.

Abbreviations: BP, blood pressure; DRV, dietary reference value; HbA1c, glycated Hb; NP, nutrient profile; SBP, systolic BP; T2D, type 2 diabetes; TEI, total energy intake. 
The NP model has been previously validated in relation to food and diet quality ${ }^{(29-33)}$, but to our knowledge no other studies have assessed the NP model in relation to cardiometabolic health in a UK population and to adherence to the UK dietary guidelines. The UK guidelines originates from the UK dietary reference values (DRV) ${ }^{(34-36)}$, which were reviewed in 2017 by Public Health England in relation to prevention of CVD in a UK population $^{(24)}$. The review highlighted a recommended daily intake of nutrients: total carbohydrate, fibre, $\mathrm{Na}$, added sugar, total fat, SFA and food groups: fruit/vegetables and weekly intake of fish (online Supplementary Table S1). The recommendations are estimated for a healthy adult British population with a mean energy intake (excluding energy from alcohol) of $8368 \mathrm{~kJ}$ (2000 kcal) for women and $10460 \mathrm{~kJ}(2500 \mathrm{kcal})$ for men.

The Airwave Health Monitoring study is currently the largest study worldwide on police force employees ${ }^{(37)}$. Recruitment commenced in 2004 across all fifty-four police forces in Great Britain. Since 2007 dietary data has been collected from participants using 7-d food diaries making it a unique cohort to investigate diet related non-communicable disease risk in a large UK young adult population. The overall aims of this study are to: (i) develop a UK specific diet quality score based on adherence to the dietary guidelines stated in the UK Dietary Policy for the Prevention of $\mathrm{CVD}^{(24)}$, that is based on selected components of UK DRV (index score) and (ii) determine the association between the DRV index and the previously established NP model with a comprehensive range of cardiometabolic risk markers among a British adult population.

\section{Methods}

\section{Study design}

This is a cross-sectional analysis of data collected as part of the Airwave Health Monitoring study ${ }^{(37)}$.

\section{Study population and ethics}

Members of the police force in Great Britain were eligible for inclusion. Further details of the recruitment procedures and data collection methods have been described elsewhere ${ }^{(37)}$. This study comprises of 5848 participants who took part in the health screen and provided dietary data between 2007 and 2012. Participants provided written informed consent and the study had ethics approval from the National Health Service Multi-Site Research Ethics Committee (MREC/13/NW/0588).

\section{Measurements of metabolic health}

The health screenings were carried out in dedicated Airwave Health Monitoring Study clinics using a standard protocol. Trained nurses conducted all clinical examinations.

Blood pressure. Sitting BP was taken using the Omron HEM 705-CP digital BP monitor (Omron Health Care). Three measurements were taken $30 \mathrm{~s}$ apart and the average was used.

Anthropometry. Participants were measured in light clothing and without shoes or socks. Height and sitting height were measured using a Marsden H226 portable stadiometer (Marsden Group) and weight using a Marsden digital weighing scale. Two measurements are taken and the average was used. Waist circumference was measured between the lower rib margin and the iliac crest in the mid-axillary line using a Wessex-finger/joint measure tape (Seca 201; Seca). Two measurements were taken and the average was used.

Blood samples. All samples were taken non-fasting. Tests were performed using serum sample except for glycated $\mathrm{Hb}$ (HbA1c) determination, which was performed using whole EDTA blood sample and glucose determination, which was performed using fluoride/oxalate sample tube. Samples were measured using an IL 650 analyser (Instrumentation Laboratory).

\section{Socio-demographic, health and lifestyle data}

Socio-demographic, health and lifestyle data were collected via a self-administrated electronic questionnaire, which the participant filled in during their clinic visit. Variables used for the present study included; age, sex, education level, ethnicity, smoking status, diagnosed diseases, medication usage and physical activity. Physical activity was measured using the International Physical Activity Questionnaire short version and the metabolic equivalent minutes per week were calculated for each participant and categorised; high (at least $60 \mathrm{~min} / \mathrm{d}$ of at least moderate-intensity activity), medium (at least $30 \mathrm{~min} / \mathrm{d}$ of at least moderate-intensity activity), low (no activity is reported or less then medium category) ${ }^{(38)}$.

\section{Dietary data}

Dietary intake was assessed with a 7-d food diary (estimated weight) using instructions from food portion photographs and common household measures as well as a general question sheet to help validate the recorded intake. The diary used has been previously validated in a larger UK epidemiological study ${ }^{(39)}$. The diaries were completed and returned via post or given to the clinic during the respondent's health screening. They were analysed using Dietplan (version 6.0; Forestfield Software), which used the UK nutrient database based on McCance and Widdowson's 'The Composition of Foods' published by the UK Food Standards Agency (2008, 6th edition). A study specific operational manual and quality auditing protocol was designed for coding and quality control of the food diaries ${ }^{(40)}$. The dietary data were checked for energy intake misreporting using the Goldberg method ${ }^{(41)}$ with the application of physical activity levels based on reported metabolic equivalents. The methods and results of under-reporting in this cohort have been previously reported in detail ${ }^{(40)}$. Sensitivity analysis was conducted to assess potential bias to the analyses (online Supplementary Table S2).

\section{Dietary reference values index dietary score computation}

The sixteen-point DRV index is a priori score reflecting adherence to Public Health England UK dietary policy for optimal health and prevention of $\mathrm{CVD}^{(24)}$, which are components derived from of the UK DRV ${ }^{(42)}$ (online Supplementary Table S1). The DRV index score was based on the intake of the six nutritional components (total carbohydrates, sugars, total fat, SFA, salt, dietary fibre) and two food group components (fruit and vegetables combined, and total fish), as listed in the UK Dietary Policy for the Prevention of 
Table 1. UK Dietary Reference Values Index scoring system for nutrient and food group mean intake per day as recommended by UK dietary reference values $^{(42)}$ and guidelines for optimal health and prevention of cardiometabolic risk ${ }^{(24)}$

\begin{tabular}{lcccccccc}
\hline Points $^{*}$ & CHO $(\%) \dagger$ & Fibre $(\mathrm{g} / \mathrm{d}) \ddagger$ & F\&V $(\mathrm{g} / \mathrm{d})$ & Fish $(\mathrm{g} / \mathrm{d}) \S$ & $\mathrm{Na}(\mathrm{mg} / \mathrm{d})$ & Fat $(\%) \dagger$ & SFA (\%) $\dagger$ & Sugar $(\%) * \|$ \\
\hline 0 & $<47.5$ & $<17.10$ & $<380$ & $<38$ & $>2520$ & $>36.75$ & $>11.55$ & $>11.55$ \\
1 & $>47.5$ to $<52.5$ & $>17.10$ to $<18.9$ & $>380$ to $<420$ & $>38$ to $<42$ & $<2520$ to $>2280$ & $<36.75$ to $>33.25$ & $<11.55$ to $>10.45$ & $<11.55$ to $>10.45$ \\
2 & $>52.5$ & $>18.9$ & $>420$ & $>42$ & $<2280$ & $<33.25$ & $<10.45 \quad$ & $<10.45 \quad$ \\
\hline
\end{tabular}

F\&V, fruit and vegetables; $\mathrm{CHO}$, carbohydrates; DRV, dietary reference values.

* 1 point: mean dietary intake is within $+/-2$ SD of the DRV criteria, 0 point: exceed 2 SD of the DRV criteria (worse diet), 2 points: exceed 2 SD of the DRV criteria (better diet).

$\dagger \%$ daily mean energy intake excluding alcohol.

$\ddagger$ NSP fibre.

$\S$ Total fish including oily fish.

II Non-milk extrinsic sugars.

CVD report. Due to the limitations of the UK nutrient database the DRV for NSP fibre was used rather than Association of Official Analytical Chemists and added sugar replaced with non-milk extrinsic sugar (NMES). Salt intake is reported as sodium (salt = $\mathrm{Na} \times 2.5)$. The DRV index score was limited to those stated in the UK Dietary Policy for the Prevention of $\mathrm{CVD}^{(24)}$ and therefore did not include all UK healthy eating recommendations. The construction of the DRV index scoring system was based on a previously reported method ${ }^{(29,43,44)}$. The mean daily intakes of the eight components were assessed according to the UK DRV and scored accordingly; 1 point represents $+/-2 \mathrm{SD}$ of DRV criteria, 0 points if intake was worse than DRV criteria and 2 points if intake was better than DRV criteria (Table 1). The points are summed to calculate an overall score between 0 and 16 points, with a higher score indicating a more favourable diet. The score was calculated from the dietary intakes of all food and drink consumed except alcohol, which was analysed separately and adjusted for with other known confounders.

\section{Nutrient profile score computation}

The construction of the NP score has been described in detail elsewhere $^{(29,44,45)}$. In brief, foods and drinks (except alcohol) score points based on their content of negative nutrients: energy, SFA, total sugar and $\mathrm{Na}$ and positive nutrients: fruit, vegetables and nut content, fibre and protein. The nutrients thresholds are derived from the UK DRV ${ }^{(46,47)}$. One point for each nutrient corresponds to $3.75 \%$ of the DRV. Each food item is given an individual score (per $100 \mathrm{~g}$ ) which then is energy adjusted (nutrient density) using previously method ${ }^{(28,29,44)}$ :

(Each food item individual score (per $100 \mathrm{~g}) \times$ energy from the food item) / TEI,

where TEI is total energy intake.

A total NP score (energy adjusted) is added up on all the foods and drinks NP (energy adjusted) to provide a daily average score:

Total NP score $=\Sigma \mathrm{NP}($ energy adjusted $)$ scores /

number of days in the dairy.

An additional algorithm ${ }^{(44)}$ is applied to the daily average NP score to scale it from 1 to 100 points:

$$
\text { Scaled NP score }=(-2) \times \text { total NP score }+70 \text {. }
$$

The interpretation of the score is a higher score indicates a diet high in food quality ('healthy' food and drinks).

\section{Clinical definitions}

Cardiometabolic risk factors and outcomes were defined by the unified international criteria for the MetS previously described with study adaptations ${ }^{(3)}$. Elevated BP was defined by systolic $\mathrm{BP}(\mathrm{SBP}) \geq 130 \mathrm{mmHg}$ and diastolic BP (DBP) $\geq 85 \mathrm{mmHg}$ or on anti-hypertensive medication. Low serum HDL-cholesterol men $\leq 1.0 \mathrm{mmol} / 1$, women $\leq 1.3 \mathrm{mmol} / 1$ or on lipid-lowering medication. TAG was excluded (not available in the study). Elevated blood sugar was defined by $\mathrm{Hb} 1 \mathrm{Ac} \geq 5.7 \%$ or on glucose-controlling medication ${ }^{(48)}$. Abdominal obesity was defined on European population waist circumference men $\geq$ $94 \mathrm{~cm}$, women $\geq 80 \mathrm{~cm}^{(3)}$. BMI were defined as weight divided by the square of height in metres. BMI categories underweight $\left(\mathrm{BMI}<18.5 \mathrm{~kg} / \mathrm{m}^{2}\right)$, normal $\left(18.5-24.9 \mathrm{~kg} / \mathrm{m}^{2}\right)$, overweight $\left(25-29.9 \mathrm{~kg} / \mathrm{m}^{2}\right)$ and obese $\left(\geq 30 \mathrm{~kg} / \mathrm{m}^{2}\right)^{(49)}$. CVD and T2D outcomes were defined as per The National Institute for Health and Care Excellence guidelines. Dyslipidaemia was defined by total cholesterol:HDL ratio cut-off $\geq 4.5 \mathrm{mmol} / \mathrm{l}$, diagnosed or on cholesterol-lowering medication ${ }^{(50)}$. Hypertension was defined by SBP $\geq 90 \mathrm{mmHg}$ and $\mathrm{DBP} \geq 140 \mathrm{mmHg}$, diagnosed or on anti-hypertensive medication. T2D was defined as $\mathrm{HbA} 1 \mathrm{c} \geq 6.5 \%$, diagnosed or on glucose-controlling medication $^{(51,52)}$.

\section{Statistical analysis}

Baseline socio-demographic and lifestyle characteristics of participants were compared between sex of study sample using Student's $t$ tests and $\chi^{2}$ as appropriate. Effect modifications by sex were examined for all analyses and the stratified results found no differences between observed associations and metabolic markers. To obtain better statistical power in the analyses men and women were combined in the study. All variables were normally distribution except alcohol, which was categorised based on revised UK recommended allowances $^{(53)} ; 2$ units/d (no alcohol, below or within, above). Other categorical variables included sex (male, female), smoking (never, current, former), and physical activity (low, moderate, high).

Diet scores association with the mean intake of dietary components included and not included (whole grains, sugar sweetened beverages, red meat, low-fat dairy products, alcohol, MUFA and PUFA) in the calculation of the dietary scores were assessed using general linear models adjusted for sex and mean 
energy intake, testing linear trend across quartiles. The association between diet scores with metabolic risk and adiposity markers (HbA1c, total cholesterol, HDL-cholesterol, BP, waist circumference, BMI) were analysed via multivariate general linear models. Association with metabolic outcome (MetS classification - 'yes'/'no') were analysed via logistic regression models. All analyses were adjusted for covariates; age, sex, BMI (except adiposity markers analysis), mean alcohol intake, smoking, physical activity, education level, dependent variable specific diagnosis and treatments. Analyses were adjusted for energy intake using the nutrient density method in both the diet scores rather than the residual or partition method, as this is the method previously applied in both the NP model and the UK dietary guidelines (i.e. DRV macronutrient intakes are reported as a proportion of TEI). Sensitivity analysis was tested on the diet scores association with cardiometabolic markers stratified by participants classified by Goldberg method as: (i) acceptable energy reporters ( $n$ 2815) and (ii) energy under-reporters ( $n$ 2721) (online Supplementary Table S2). Participants on weight loss diet and over energy reporters were excluded from the analysis.

SAS 9.3 (SAS Institute Inc.) was used for all analysis and statistical significance was set at $P<0.05$.

\section{Results}

\section{Demographic and lifestyle characteristics of the sample}

Table 2 shows the descriptive statistics of the sample ( $n$ 5848) across the DRV quartiles. The characteristics of healthy eaters

Table 2. Demographic and lifestyle characteristics across dietary reference values (DRV) index quartiles (Q), the Airwave Health Monitoring Study ( $n$ 5848)

(Mean values with their standard errors; percentages)

\begin{tabular}{|c|c|c|c|c|c|}
\hline Variables & Q1 (least healthy) & Q2 & Q3 & Q4 (healthiest) & \\
\hline Range & $(0-1.99)$ & $(2.00-3.99)$ & $(4.00-6.99)$ & $(7 \cdot 00-16 \cdot 00)$ & $P_{\text {trend }}{ }^{*}$ \\
\hline$n$ & 1758 & 1447 & 1098 & 1544 & \\
\hline $\operatorname{Sex}(\%)$ & & & & & $<0.0001$ \\
\hline Female & $25 \cdot 13$ & $25 \cdot 72$ & 19.69 & 29.44 & \\
\hline Men & 33.41 & 24.08 & $18 \cdot 16$ & $24 \cdot 23$ & \\
\hline Age (years) & & & & & 0.002 \\
\hline Mean & $40 \cdot 88$ & $40 \cdot 77$ & $41 \cdot 14$ & 41.85 & \\
\hline SE & 0.23 & 0.23 & 0.22 & 0.24 & \\
\hline Education level (\%) & & & & & 0.04 \\
\hline Post-graduate & 3.87 & 3.66 & $5 \cdot 19$ & 4.53 & \\
\hline Bachelor degree & 31.74 & 28.40 & $26 \cdot 68$ & 30.89 & \\
\hline A-level & 8.42 & 7.33 & 7.56 & $5 \cdot 76$ & \\
\hline Vocational & 33.45 & 33.72 & $32 \cdot 33$ & 29.07 & \\
\hline GCSE/O level & $16 \cdot 78$ & $20 \cdot 66$ & 21.68 & 22.09 & \\
\hline No formal qualification & 5.75 & $6 \cdot 22$ & 6.56 & 6.93 & \\
\hline Alcohol $(g / d)$ & & & & & $<0.0001$ \\
\hline Mean & $15 \cdot 28$ & 14.80 & $13 \cdot 22$ & 11.66 & \\
\hline SE & 0.38 & 0.41 & 0.37 & 0.40 & \\
\hline No alcohol (\%) & $17 \cdot 85$ & $20 \cdot 22$ & $19 \cdot 87$ & $25 \cdot 19$ & $<0.0001$ \\
\hline Within guidelines (\%) & 44.70 & $42 \cdot 65$ & $46 \cdot 28$ & $46 \cdot 64$ & \\
\hline Above guidelines (\%) & 37.45 & $37 \cdot 13$ & 33.85 & $28 \cdot 17$ & \\
\hline Cigarette smoking (\%) & & & & & $<0.0001$ \\
\hline Never & 67.03 & 67.86 & $71 \cdot 13$ & 71.63 & \\
\hline Former & 22.40 & $22 \cdot 81$ & 22.04 & $23 \cdot 19$ & \\
\hline Current & 10.57 & 9.33 & 6.83 & $5 \cdot 18$ & \\
\hline Physical active (\%) & & & & & $<0.0001$ \\
\hline Low (<600 min/week) & 12.96 & $10 \cdot 30$ & $9 \cdot 20$ & $8 \cdot 81$ & \\
\hline Moderate (>600 min/week) & $40 \cdot 42$ & $42 \cdot 43$ & 42.53 & 38.08 & \\
\hline High (>3000 min/week) & $46 \cdot 62$ & $47 \cdot 27$ & $48 \cdot 27$ & $53 \cdot 11$ & \\
\hline BMI $\left(\mathrm{kg} / \mathrm{m}^{2}\right)$ & & & & & 0.0005 \\
\hline Mean & $27 \cdot 01$ & 26.95 & $26 \cdot 74$ & $26 \cdot 57$ & \\
\hline SE & 0.10 & 0.10 & 0.10 & 0.11 & \\
\hline Normal (18.5-24.99) (\%) & $29 \cdot 62$ & 32.69 & 34.88 & 35.62 & $<0.0001$ \\
\hline Overweight (25-29.99) (\%) & 48.55 & $45 \cdot 82$ & $45 \cdot 54$ & $46 \cdot 89$ & \\
\hline Obese $(>30)(\%)$ & 21.83 & 21.49 & 19.58 & $17 \cdot 49$ & \\
\hline Waist circumference $(\mathrm{cm})$ & & & & & $<0.0001$ \\
\hline Mean & $90 \cdot 71$ & $89 \cdot 30$ & 88.62 & 87.63 & \\
\hline SE & 0.36 & 0.31 & 0.35 & 0.30 & \\
\hline \multicolumn{6}{|l|}{ CVD (\%)† } \\
\hline Hypertension & 32.58 & $30 \cdot 89$ & $30 \cdot 60$ & 31.35 & 0.43 \\
\hline Dyslipidaemia & 26.95 & $25 \cdot 02$ & 24.41 & $23 \cdot 77$ & 0.03 \\
\hline Diabetes type 2 & 4.49 & 3.39 & 3.37 & $3 \cdot 17$ & 0.05 \\
\hline
\end{tabular}

Q1, first quartile; Q2, second quartile; Q3, third quartile; $Q 4$, fourth quartile.

* $P$ value linear model (continuous variable) Mantel-Haenszel $x^{2}$ test (categorical variables).

† Self-reported or on specific health outcome treatment. 
Table 3. Mean intake of nutritional components across diet scores quartiles $(\mathrm{Q}) 1$ (least healthy) and 4 (healthiest) adjusted for sex and energy in Airwave Health Monitoring Study ( $n$ 5848)

(Means adjusted for sex and mean energy intake)

\begin{tabular}{|c|c|c|c|c|c|c|}
\hline & \multicolumn{2}{|c|}{ DRV score } & \multirow[b]{2}{*}{$P^{\star}$} & \multicolumn{2}{|c|}{ NP score } & \multirow[b]{2}{*}{$P^{\star}$} \\
\hline & Q1 & Q4 & & Q1 & Q4 & \\
\hline \multicolumn{7}{|l|}{ Nutrients } \\
\hline Energy intake $(\mathrm{kJ} / \mathrm{d})$ & 7749 & 7134 & $<0.0001$ & 8192 & 6180 & $<0.0001$ \\
\hline Energy intake $(\mathrm{kcal} / \mathrm{d})$ & 1852 & 1705 & & 1958 & 1477 & $<0.0001$ \\
\hline Alcohol $(\mathrm{g} / \mathrm{d})$ & 15 & 12 & $<0.0001$ & 13 & 14 & 0.23 \\
\hline Protein $(\mathrm{g} / \mathrm{d}) \dagger$ & 81 & 80 & 0.3 & 81 & 79 & 0.03 \\
\hline Fat $(g / d) \dagger$ & 81 & 57 & $<0.0001$ & 87 & 54 & $<0.0001$ \\
\hline SFA $(g / d) \dagger$ & 30 & 19 & $<0.0001$ & 34 & 18 & $<0.0001$ \\
\hline MUFA (g/d) & 28 & 21 & $<0.0001$ & 28 & 22 & $<0.0001$ \\
\hline PUFA (g/d) & 14 & 12 & $<0.0001$ & 13 & 12 & $<0.0001$ \\
\hline Carbohydrate $(\mathrm{g} / \mathrm{d}) \dagger$ & 203 & 244 & $<0.0001$ & 231 & 206 & $<0.0001$ \\
\hline Fibre $(g / d) \dagger \ddagger$ & 11 & 17 & $<0.0001$ & 12 & 15 & $<0.0001$ \\
\hline Total sugar $(\mathrm{g} / \mathrm{d}) \dagger$ & 76 & 105 & $<0.0001$ & 95 & 82 & $<0.0001$ \\
\hline $\mathrm{Na}(\mathrm{mg} / \mathrm{d}) \dagger$ & 2930 & 2652 & $<0.0001$ & 2996 & 2470 & $<0.0001$ \\
\hline \multicolumn{7}{|l|}{ Food groups } \\
\hline Fruit and vegetables $(\mathrm{g} / \mathrm{d}) \dagger$ & 222 & 461 & $<0.0001$ & 256 & 395 & $<0.0001$ \\
\hline Whole grains $(g / d)$ & 32 & 68 & $<0.0001$ & 34 & 61 & $<0.0001$ \\
\hline Fish $(\mathrm{g} / \mathrm{d})$ & 13 & 33 & $<0.0001$ & 18 & 28 & $<0.0001$ \\
\hline Oily fish $(\mathrm{g} / \mathrm{d})$ & 7 & 21 & $<0.0001$ & 10 & 17 & $<0.0001$ \\
\hline Low-fat dairy products $(\mathrm{g} / \mathrm{d})$ & 168 & 228 & $<0.0001$ & 171 & 214 & $<0.0001$ \\
\hline Red meat $(g / d)$ & 80 & 51 & $<0.0001$ & 71 & 57 & $<0.0001$ \\
\hline Processed meat $(\mathrm{g} / \mathrm{d})$ & 42 & 26 & $<0.0001$ & 42 & 25 & $<0.0001$ \\
\hline Sweet and sugary beverages $(\mathrm{g} / \mathrm{d})$ & 61 & 59 & 0.6 & 71 & 44 & $<0.0001$ \\
\hline \multicolumn{7}{|l|}{ Diet quality score } \\
\hline DRV score & & & & 2 & 7 & $<0.0001$ \\
\hline NP score & 54 & 60 & $<0.0001$ & & & \\
\hline
\end{tabular}

DRV, dietary reference value index; NP, nutrient profile model.

* $P$ value for linear trend across diet score quartiles.

$\dagger$ Nutrients and food groups included in the diet scores.

$\ddagger$ NSP fibre.

are more likely to be women and have a healthier lifestyle: drink less alcohol and exercise more. Healthier eaters had a lower BMI, waist circumference and prevalence of dyslipidaemia and T2D (unadjusted). Characteristics by sex are presented in the online Supplementary Table S3.

\section{Association between diet scores and nutritional} components

Table 3 shows that across the DRV index quartiles there is a strong trend for a consumption of other 'favourable' nutritional components both single nutrients and food groups and a reverse association with 'unfavourable' ones except for total sugar (variable includes sugar from fruit and fruit juices). Similar trends are observed across the NP score except no association is seen with alcohol intake. The diet scores are also associated with each other $\left(P_{\text {trend }}<0 \cdot 0001\right)$, Spearman's partial correlation coefficient $0 \cdot 64, P<0 \cdot 0001$ adjusted for sex and age (results not shown in table).

\section{Association between diet scores and cardiometabolic markers}

A higher NP score (per two-point increase) was associated with HbA1c $(\beta-0.01 \%, P<0.0001)$, total cholesterol $(\beta-0.33 \mathrm{mmol} / \mathrm{l}$, $P<0.0001)$ and an increase in BMI $\left(\beta 0.06 \mathrm{~kg} / \mathrm{m}^{2}, P=0.01\right)$ (Table 4). The DRV score (per two-point increase) was inversely associated with HbA1c $(\beta-0.02 \%, P=0.003)$, total cholesterol ( $\beta-0.06 \mathrm{mmol} / \mathrm{l}, P<0.0001)$, HDL-cholesterol $(\beta-0.01 \mathrm{mmol} / \mathrm{l}$, $P=0.001)$, BMI $\left(\beta-0.15 \mathrm{~kg} / \mathrm{m}^{2}, P<0.0001\right)$ and waist circumference $(\beta-0.56 \mathrm{~cm}, P<0.0001)$ (Table 4$)$. No significant associations were found with SBP and DBP with any of the diet scores. Standardised coefficients for both scores are presented in the online Supplementary Table S4.

\section{Association between diet scores and cardiometabolic outcomes}

Individuals with a higher NP score (more favourable diet) were less likely to have elevated blood sugar $(\mathrm{OR}=0 \cdot 98, P=0 \cdot 001)$. $\mathrm{NP}$ was not associated with CVD hypertension and dyslipidaemia or T2D (Table 5).

DRV score was associated with a reduced risk for several metabolic outcomes (Table 5). Individuals with a higher DRV score (more favourable diet) were less likely to have T2D (OR $0.94, P=0.01$ ), elevated blood sugar (OR 0.97, $P=0.01$ ), abdominal obesity (OR 0.94, $P<0.0001$ ) and obesity (OR 0.95 , $P<0 \cdot 0001)$. No association were seen with DRV score and CVD hypertension and dyslipidaemia.

\section{Sensitivity analysis}

Sensitivity analysis was carried out to test if bias may have been introduced by an element of participants' energy misreporting 
Table 4. Associations between diet scores and cardiometabolic risk markers in Airwave Health Monitoring Study $(n$ 5848) ( $\beta$-Coefficients with their standard errors)

\begin{tabular}{|c|c|c|c|c|c|c|}
\hline & \multicolumn{3}{|c|}{ DRV score } & \multicolumn{3}{|c|}{ NP score } \\
\hline & $\beta^{*}$ & SE & $P$ & $\beta^{*}$ & SE & $P$ \\
\hline HbA1c (\%) & -0.02 & 0.004 & 0.003 & -0.01 & 0.003 & $<0.0001$ \\
\hline $\mathrm{SBP}(\mathrm{mmHg})$ & 0.22 & 0.118 & 0.05 & 0.12 & 0.076 & 0.17 \\
\hline $\mathrm{DBP}(\mathrm{mmHg})$ & -0.10 & 0.080 & 0.28 & -0.01 & 0.052 & 0.72 \\
\hline HDL-cholesterol (mmol/l) & -0.01 & 0.003 & 0.001 & -0.003 & 0.002 & $0 \cdot 10$ \\
\hline Total cholesterol (mmol/li) & -0.06 & 0.008 & $<0.0001$ & -0.33 & 0.050 & $<0.0001$ \\
\hline Waist circumference $(\mathrm{cm}) \dagger$ & -0.56 & 0.092 & $<0.0001$ & 0.05 & 0.060 & 0.41 \\
\hline BMI $\left(\mathrm{kg} / \mathrm{m}^{2}\right) \ddagger$ & -0.15 & 0.036 & $<0.0001$ & 0.06 & 0.02 & 0.01 \\
\hline
\end{tabular}

Table 5. Associations between diet scores and cardiovascular risk and diabetes in Airwave Health Monitoring Study $(n 5848)$ (Odds ratios and $95 \%$ confidence intervals)

\begin{tabular}{|c|c|c|c|c|c|c|c|}
\hline & \multirow[b]{2}{*}{ Cases/subcohort } & \multicolumn{3}{|c|}{ DRV score } & \multicolumn{3}{|c|}{ NP score } \\
\hline & & $\mathrm{OR}^{*}$ & $95 \% \mathrm{Cl}$ & $P$ & $\mathrm{OR}^{*}$ & $95 \% \mathrm{Cl}$ & $P$ \\
\hline \multicolumn{8}{|c|}{ Cardiovascular risk and diabetes } \\
\hline Hypertension & $1840 / 4008$ & 1.01 & $0.99,1.03$ & 0.52 & 1.01 & $1.00,1.03$ & 0.06 \\
\hline Dyslipidaemia & $1471 / 4377$ & 0.99 & $0.97,1.02$ & 0.64 & 1.01 & $0.99,1.03$ & 0.07 \\
\hline Type 2 diabetes & $214 / 5848$ & 0.94 & $0.90,0.99$ & 0.01 & 0.98 & $0.95,1.01$ & 0.25 \\
\hline \multicolumn{8}{|c|}{ Metabolic syndrome risk factors $†$} \\
\hline Increased blood pressure & $3015 / 2833$ & 1.01 & $0.99,1.03$ & 0.31 & 1.02 & $1.00,1.03$ & 0.03 \\
\hline Low HDL-cholesterol & $872 / 4976$ & 1.04 & $1.01,1.06$ & 0.006 & 1.03 & $1.01,1.05$ & 0.002 \\
\hline Increased blood sugar & $2408 / 3440$ & 0.97 & $0.96,0.99$ & 0.01 & 0.98 & $0.97,0.99$ & 0.001 \\
\hline Obesity $\ddagger$ & $1180 / 4669$ & 0.95 & $0.93,0.98$ & $<0.0001$ & 1.00 & $0.99,1.02$ & 0.57 \\
\hline Abdominal obesity§ & $2934 / 2914$ & 0.94 & $0.92,0.96$ & $<0.0001$ & 1.00 & $1.00,1.01$ & 0.96 \\
\hline
\end{tabular}

DRV, dietary reference values; NP, nutrient profile.

* Logistic regression models represent the increase in health outcome per 1-point increase in the diet score, adjusted for age, sex, BMI, alcohol, smoking, physical activity and education level.

† Based on metabolic syndrome risk classification

$\ddagger$ Not adjusted for BMI.

$\S$ Adjusted for height not BMI.

(online Supplementary Table S2). The stratified analysis showed that the reverse association between DRV score SBP, and HDL-cholesterol was NS in acceptable energy reports only in energy under-reporters. No other modification was observed for DRV score and the other metabolic risk markers. The stratified analysis only showed modified association between NP score, BMI and waist circumference. The stratified analyses showed the NP score association with BMI was reverse in both groups (energy reporters and energy under reporters). Furthermore, NP scores relationship with waist circumference inversed in both these groups. In addition, sensitivity analyses were conducted for cardiometabolic health outcomes in logistic regression models excluding energy misreporting no difference were observed (result tables not shown).

\section{Discussion}

This cross-sectional study demonstrates that both diet scores NP model and DRV index are associated with over nineteen other dietary components essential to assess a person's diet quality including nutritional components included in the UK dietary guidelines and Eat-Well guide. Similar results have been previously shown with the NP model ${ }^{(17,29,33,54)}$, suggesting that diet nutrient based scores may capture intake of a number of other important food groups in a person's diet. The 'healthiest diets' (DRV quartile 4) consumed a mean daily energy intake of $7134 \mathrm{~kJ}$ (1705 kcal), total fat $57 \mathrm{~g}$ (30\% TEI), SFA $19 \mathrm{~g}$ (10\% TEI), carbohydrate $244 \mathrm{~g}$ ( $57 \% \mathrm{TEI})$ and $461 \mathrm{~g}$ of fruit/vegetables, which are in line with the national dietary guidelines ${ }^{(34-36,53)}$ except for lower intake of NSP fibre ( $17 \mathrm{~g} v$. recommendation of $24 \mathrm{~g} / \mathrm{d}$ ). Similar results were seen for NP score. The results support the application of both scores in epidemiological studies to capture intake of essential nutrients and food groups in a UK diet. However, these results are based on a specific study population ( $95 \%$ white British) who are younger (mean age 41 years) and primary men (60\%) (online Supplementary Table S3). Therefore, the 'healthiest diets' identified in this study based on these diet scores does not necessarily represent a random sample from the general UK population. The scores are based on specific components from the UK dietary guidelines, which may not capture all food groups in a UK diet. 
Furthermore, such country-specific diet scores may not capture essential food and nutrient groups commonly consumed in other countries. Similar limitations to diet scores have been previously discussed by Kant $^{(14)}$ and Moeller et al. ${ }^{(14,26)}$. However, in this study population both scores, which only including eight dietary components demonstrated association with nineteen dietary components.

The DRV score was inversely associated with HbA1c, total cholesterol and adiposity markers (BMI and waist circumference). These relationships were driven by diets higher in fruit, vegetables, fibre and lower in sugar, $\mathrm{Na}$, fat and SFA (online Supplementary Table S5), which have also been demonstrated in other single nutrient studies and $\mathrm{RCT}^{(8,12,55,56)}$. The Cardiovascular disease risk Reduction trial (CRESSIDA) ${ }^{(56)}$ also showed that an intervention diet adhering to the UK dietary guidelines measured by Na, total fat, SFA, NMES, fruit/ vegetables and whole grains lowered BMI, BP and lipid profile in a UK study population. The intervention trial provided diet education based on food groups from the 'Eat-Well Guide', which are food recommendations based on the UK dietary guidelines. The CRESSIDA study highlighted that six nutrients and one food group could be used to measure and reflect the intake of a wide range of foods from 'Eat-Well Guide'. Similar results were shown previously with the NP score and a wide range of foods from 'Eat-Well Guide ${ }^{,(33)}$. Our study also showed that both diet scores were additionally associated with five different dietary components which were not included in the scores but considered essential to a healthy diet (lower intakes of red meat, processed meat and alcohol and higher intakes of low-fat dairy products and whole grains). This suggests that the diet scores may serve as an efficient dietary scoring method in epidemiological studies.

NP score only showed inverse association HbA1c and total cholesterol. In contrary, NP was associated with a higher BMI. However, stratified sensitivity analysis of NP association with BMI showed that the association was reversed in both acceptable energy reporters and energy under-reporters. These findings are challenging to compare as only one other study, the Supplementation en Vitamines et Mineraux Antioxydants (SUVIMAX) cohort, to our knowledge, has investigated an adaptation of NP model in relation to cardiometabolic risk factors in a French population ${ }^{(57)}$. The SUVIMAX cohort saw no association with waist circumference, fasting glucose, or blood lipids. Another study by Arambepola et $a l^{(33)}$ also did not report any correlation with NP model (energy adjusted) and BMI. The NP model's limitations have been discussed by several authors $^{(29,32,33,47)}$. The main limitation is that NP model only measure one aspect of diet quality (food quality and density) and it does not measure overall nutritional intake, diet patterns or variety of foods consumed. Another limitation may be the method applied to nutrient density of the score. There exist various methods of energy adjusting diet scores such as residual and partition methods. This study chose to apply a nutrient density (energy adjusted) method previously applied in other studies of the NP model ${ }^{(29,32,33)}$. However, such nutrient density methods may be limited in capturing healthiness in certain food groups such as olive oils and fatty fish ${ }^{(32,58)}$. Furthermore, it may be argued that if energy intake lies in the causal pathway between certain nutrient groups, for example high fat and sugar and cardiometabolic outcomes, it should be treated as a mediator rather than a confounder.

The DRV score inverse association with HDL-cholesterol was not significant in acceptable energy reporters suggesting that the association may have been biased by some element of misreporting of dietary intake. Neither of the diet scores (DRV or NP) showed a positive association HDL-cholesterol. Single nutrient analyses (online Supplementary Table S5) showed that dietary variables carbohydrate, sugar and fat, which are incorporated in both the scores was driving an inverse association with HDL-cholesterol. Both diet scores also showed a significant lower intake of PUFA and MUFA across all quartiles. Suggesting that neither of them captured essential nutrients in the diet which are positively associated with HDL-cholesterol such as PUFA and MUFA ${ }^{(8,12)}$. This may explain why no positive relationship was observed, as reported for DASH and MDS, which include these nutrients ${ }^{(13,59)}$.

This study also reported an increase in SBP with a higher DRV score. However, this association was no longer significant in the stratified sensitivity analysis in acceptable energy reporters, only in energy under-reporters. Suggesting some introduction of bias in misreporting of nutrients or foods high in $\mathrm{Na}$, for example processed foods in this group.

The study also showed NP score had reverse relationship with elevated blood sugar, which was driven by its relationship with HbA1c. No other associations were observed for cardiometabolic outcomes as seen with other NP models ${ }^{(54,57,60)}$. SUVIMAX cohort found a lower UK NP score (adapted version) was associated with the MetS (OD 1.06) ${ }^{(57)}$. The USA NP model found a higher score (healthier food quality) was associated with a lower risk of T2D, CVD and mortality rates in participants from the Nurses' Health Study and Health Professionals FollowUp Study ${ }^{(60)}$.

Our study also observed that participants classified as consuming a healthier diet (higher DRV score) were less likely to be obese, abdominal obese and to have T2D. These results were predominately driven by the DRV inverse relationship with BMI, waist circumference and HbA1c, which are in line with previous studies of other diet quality scores ${ }^{(14)}$. Nicklas et al. reported similar inverse relationships and OR with metabolic risk factors elevated blood sugar, abdominal obesity and obesity was by reported in US National Health and Nutrition Examination Survey for individuals aligned with the national diet guidelines ${ }^{(16)}$.

This study highlights some of the limitations of the NP model in relation to capturing diet quality and associated cardiometabolic health outcomes. The nutrient composition of individual foods is not the only determinant of the overall nutrient composition of diets. Assessing the healthiness of diets is complex and often requires a holistic approach to capture association with health outcomes ${ }^{(14)}$. The NP model may not reflect the variety of different foods that make up the diets and the healthiness of the diets, for example dietary patterns. Therefore, assessing a single food healthiness in its own would not be expected to capture the combination of different foods and quantity needed for a balanced diet. These limitations were also discussed in detailed by Nicklas et al. ${ }^{(58)}$, which highlighted 
issues related to NP models' algorithms applied such the nutrients and food groups studied and their threshold values applied and their nutrient density score. The NP model originated for use of guiding the public health in choosing healthier foods, that is labelling 'Traffic Light System' and may therefore serve as a better tool in this context rather than capturing associations between overall healthiness of diet and health outcomes in epidemiological studies.

In summary, evidence suggests the NP model is a relevant tool to measure quality of individual foods contributing to an individual diet in a UK population. However, our study demonstrates that a diet score (DRV index) assessing overall diet quality as alignment to important components of the national dietary guidelines performs better in capturing diets relation to cardiometabolic risk, compared with a food-based score (NP model). This also supports the importance of promoting both overall dietary guidelines in the public health as well as food choices 'Eat Well Guide' and labelling 'Traffic Light System' and in relation to their beneficial roles on cardiometabolic health.

The main strength of this study is the dietary and clinical data on a relatively large sample of British adults. Data from 7-d food diary provide in-depth insights in relation to adiposity and metabolic markers, compared with usual measures of diet in cohort studies. Use of a 7-d food diary is known to limit measurement errors and provide accurate estimates of individual diet intake ${ }^{(61)}$ compared with other methods such as FFQ or $24-\mathrm{h}$ recall $^{(62,63)}$. This method also allows for an in-depth analysis of overall diet quality. Another strength is the study's rigorous quality control of the dietary data; regular coder training, a standard operational protocol and quality control audit cycle helped maintain a low mean code error, which has been described elsewhere ${ }^{(40)}$.

The first and major limitation of this study is the use of crosssectional study design, which cannot provide evidence of a causal relationship between the diet scores and metabolic risk factors. These results could be due to the reverse causality in individuals who have made diet improvements after being informed of a medical condition. Another limitation lies in the non-generalisation of the results due to those who completed the food diaries may introduce selection bias, leading to underestimation of the strength of the association. Participants volunteering to complete food diaries may be more health conscious and vary in lifestyle characteristics. However, the diet and clinical data reported in this study is comparable with the general UK population. The dietary data (mean energy and macronutrient intake) is comparable with the NDNS ${ }^{(12,64)}$. The mean daily energy intake reported in this study was $7000 \mathrm{~kJ}$ (1673 kcal) for women and $8665 \mathrm{~kJ}$ (2071 kcal) for men compared with $6527 \mathrm{~kJ}(1560 \mathrm{kcal})$ and $8502 \mathrm{~kJ}$ (2032 kcal) in the NDNS. The Airwave Health Monitoring Study population is a young cohort, which may differ from the general population in health outcomes. However, the sample's prevalence of cardiovascular risk factors (online Supplementary Table S2) is both comparable with the total cohort ${ }^{(37)}$, and representative of the general UK population (Health Survey for England 2012) ${ }^{(65)}$; hypertension ( $28 \% v$. Health Survey $29 \%$ ), obesity (20\% $v$. Health Survey $19 \cdot 5 \%$ ) and diabetes (5\% $v$. Health Survey $5 \cdot 8 \%$ ).
Only dyslipidaemia is lower in the sample $4.75 \%$, compared with two thirds of general population ${ }^{(66)}$.

Another limitation in this study is the prevalence and systematic bias of underreporting, which have been discussed elsewhere $^{(40)}$. Despite both diet scores were energy adjusted (nutrient density methods) sensitivity analysis found confounding effect of the under-reporters between the association of DRV index, HDL-cholesterol and SBP. Furthermore, differences were observed for NP model and adiposity markers BMI and waist circumference (online Supplementary Table S2). Ideally nutritional assessments and misreporting should have been investigated further by other validating methods such as doubly labelled water, urine biomarkers, for example urine $\mathrm{Na}$, $\mathrm{K}$ and $\mathrm{N}$ to help confirm accuracy of self-reported dietary data and to limit bias. However, these analyses were not available for this study.

In conclusion, this study suggests that the NP model was associated with an overall diet quality (higher DRV score) aligning with important components of UK dietary guidelines. However, the NP model is only inversely associated with total cholesterol and elevated blood sugar (HbA1c). Its relationship to other cardiometabolic risk factor remains inconclusive. Whereas, the DRV index captured important food patterns and quality, which are inversely associated with several metabolic risk factors, adiposity markers and T2D. The study supports the application of the DRV index in epidemiological studies investigating overall diet quality in relation to metabolic risk of CVD and T2D in a UK population. However, more studies, especially longitudinal studies, are needed to support these findings and to confirm the effectiveness of the DRV index on cardiometabolic health.

\section{Acknowledgements}

The authors thank all participants in the Airwave Health Monitoring Study Health Monitoring Study. The authors would also like to thank all the dietary coders who have contributed to the generation of the dietary data: Shatha Alrabiah, Jessica Ayling, Andrea Carames, Zhengyu Fan, Kirsty Frost, Louise Hirichi, Zanna Hofstede, Niamh O'Sullivan, and Yiling Zhu. The authors also thank Andy Heard, database manager, Deepa Singh, clinical lead who set up the clinics and Louisa Cavaliero who assisted in data collection and management.

The Airwave Health Monitoring Study is funded by the Home Office (grant no. 780- TETRA) with additional support from the National Institute for Health Research (NIHR), Imperial College Healthcare NHS Trust (ICHNT) and Imperial College Biomedical Research Centre (BRC). This paper presents independent research funded by the Home Office with additional support from the NIHR, ICHNT and Imperial College BRC at Imperial College Healthcare NHS Trust. The views expressed are those of the authors and not necessarily those of the Home Office, ICHNT, the BRC, the NHS, the NIHR or the Department of Health. P. E. acknowledges support from the NIHR Biomedical Research Centre at Imperial College Healthcare NHS Trust and Imperial College London, the NIHR Health Protection Research Unit on Health Effects of Environmental Hazards (HPRU-2012-10141), and the Medical Research Council-Public 
Health England (MRC-PHE) Centre for Environment and Health (MR/L01341X/1). The UK Dementia Research Institute (DRI) is funded by the Medical Research Council, Alzheimer's Society and Alzheimer's Research UK.

G. F., R. E., R. G. and Q. C. formulated the research question and methodological design; R. E. was responsible for data analysis and drafting of the manuscript. G. F., P. E. R. G. and Q. C. contributed to the interpretation of results and final manuscript. R. E., R. G., Y. M., and K. L. all contributed to the development of the food diary coding protocol, coder training and audit checking. Q. C. assisted R. E. and R. G. with the dietary data extraction and cleaning. M. A. and J. S. were responsible for the validation of the non-dietary data extracts used in the analyses. P. E. is the principal investigator of the Airwave Health Monitoring Study. All authors read and approved the final manuscript.

P. E. is a UK DRI Professor, UK DRI at Imperial College London. G. F. is an NIHR senior investigator. The authors declare that there are no conflicts of interest.

\section{Supplementary material}

For supplementary material/s referred to in this article, please visit https://doi.org/10.1017/S0007114518000016

\section{References}

1. World Health Organization (2012) Fact Sheets: Cardiovascular Diseases. Geneva: WHO. http://www.who.int/mediacentre/ factsheets/fs317/en/ (accessed October 2017).

2. Despres JP \& Lemieux I (2006) Abdominal obesity and metabolic syndrome. Nature 444, 881-887.

3. Alberti K, Eckel R, Grundy S, et al. (2009) Harmonizing the metabolic syndrome. A joint interim statement of the IDF Task Force on Epidemiology and Prevention; NHL and Blood Institute; AHA; WHF; IAS; and IA for the Study of Obesity. Circulation 120, 1640-1645.

4. Tillin T, Forouhi N, Johnston DG, et al. (2005) Metabolic syndrome and coronary heart disease in South Asians, AfricanCaribbeans and white Europeans: a UK population-based cross-sectional study. Diabetologia 48, 649-656.

5. Lawlor DA, Smith GD \& Ebrahim S (2006) Does the new International Diabetes Federation definition of the metabolic syndrome predict CHD any more strongly than older definitions? Findings from the British Women's Heart and Health Study. Diabetologia 49, 41-48.

6. Grundy SM (2007) Metabolic syndrome: a multiplex cardiovascular risk factor. J Clin Endocrinol Metab 92, 399-404.

7. Park YWZS, Palaniappan L, Heshka S, et al. (2003) The MetS: prevalence and associated risk factor findings in the US population from Third National Health and Nutrition Examination Survey, 1988-1994. Arch Int Med 163, 427-436.

8. World Health Organization (2003) Diet, Nutrition and the Prevention of Chronic Diseases, Technical Report Series. Geneva: WHO.

9. Brunner EJ, Mosdøl A, Witte DR, et al. (2008) Dietary patterns and $15-y$ risks of major coronary events, diabetes, and mortality. Am J Clin Nutr 87, 1414-1421.

10. Iqbal R, Anand S, Ounpuu S, et al. (2008) Dietary patterns and the risk of acute myocardial infarction in 52 countries: results of the INTERHEART Study. Circulation 118, 1929-1937.
11. Babio N, Bullo M \& Salas-Salvado J (2009) Mediterranean diet and metabolic syndrome: the evidence. Public Health Nutr 12, 1607-1617.

12. Committee on Medical Aspects of Food Policy (1994) Nutritional Aspects of Cardiovascular Disease. Report on Health and Social Subjects no. 46. London: Committee on Medical Aspects of Food Policy.

13. Esposito K, Kastorini CM, Panagiotakos DB, et al. (2011) Mediterranean diet and weight loss: meta-analysis of randomized controlled trials. Metab Syndr Relat Disord 9, 1-12.

14. Kant AK (2004) Dietary patterns and health outcomes. J Am Diet Assoc 104, 615-635.

15. Waijers PM, Feskens EJ \& Ocké MC (2007) A critical review of predefined diet quality scores. Br J Nutr 97, 219-231.

16. Nicklas TA, O'Neil CE \& Fulgoni VL (2012) Diet quality is inversely related to cardiovascular risk factors in adults. $J$ Nutr 142, 2112-2118.

17. Lassale C, Galan P, Julia C, et al. (2013) Association between Adherence to Nutritional Guidelines, the Metabolic Syndrome and Adiposity Markers in a French Adult General Population. PLOS ONE 8, e76349.

18. Seymour JD, Calle EE, Flagg EW, et al. (2003) Diet quality index as a predictor of short-term mortality in the American Cancer Society Cancer Prevention Study II Nutrition Cohort. Am J Epidemiol 157, 980-988.

19. Conlin PR, Chow D, Miller ER III, et al. (2000) The effect of dietary patterns on blood pressure control in hypertensive patients: results from the dietary approaches to stop hypertension (DASH) trial. Am J Hypertens 13, 949-955.

20. Hu FB (2002) Dietary pattern analysis: a new direction in nutritional epidemiology. Curr Opin Lipidol 13, 3-9.

21. Toft U, Kristoffersen LH, Lau C, et al. (2007) The Dietary Quality Score: validation and association with cardiovascular risk factors: the Inter99 study. Eur J Clin Nutr 61, 270-278.

22. Tucker KL, Hallfrisch J, Qiao N, et al. (2005) The combination of high fruit and vegetable and low saturated fat intakes is more protective against mortality in aging men than is either alone: The Baltimore Longitudinal Study of Aging. J Nutr $\mathbf{1 3 5}$, 556-561.

23. Sacks FM, Svetkey LP, Vollmer WM, et al. (2001) Effects on blood pressure of reduced dietary sodium and the Dietary Approaches to Stop Hypertension (DASH) diet. N Engl J Med 344, 3-10.

24. Levy L \& Tedstone A (editors) (2017) UK Dietary Policy for the Prevention of Cardiovascular Disease. Healthcare. London: Multidisciplinary Digital Publishing Institute.

25. Trichopoulou A, Orfanos P, Norat T, et al. (2005) Modified Mediterranean diet and survival: EPIC-elderly prospective cohort study. BMJ 330, 991.

26. Moeller SM, Reedy J, Millen AE, et al. (2007) Dietary patterns: challenges and opportunities in dietary patterns research: an Experimental Biology workshop, April 1, 2006. J Am Diet Assoc 107, 1233-1239.

27. Quinio C, Biltoft-Jensen A, De Henauw S, et al. (2007) Comparison of different nutrient profiling schemes to a new reference method using dietary surveys. Eur J Nutr $\mathbf{4 6}$, Suppl. 2, 37-46.

28. Volatier JL, Biltoft-Jensen A, De Henauw S, et al. (2007) A new reference method for the validation of the nutrient profiling schemes using dietary surveys. Eur J Nutr 46, Suppl. 2, 29-36.

29. Scarborough P, Arambepola C, Kaur A, et al. (2010) Should nutrient profile models be 'category specific' or 'acrossthe-board'? A comparison of the two systems using diets of British adults. Eur J Clin Nutr 64, 553-560. 
30. Tzoulaki I, Patel CJ, Okamura T, et al. (2012) A nutrient-wide association study on blood pressure. Circulation 126, 2456-2464.

31. World Health Organization (2010) Nutrient Profiling - Report of WHO/IASO Technical Meeting. London: WHO.

32. Masset G, Scarborough P, Rayner M, et al. (2015) Can nutrient profiling help to identify foods which diet variety should be encouraged? Results from the Whitehall II cohort. Br J Nutr 113, 1800-1809.

33. Arambepola C, Scarborough P \& Rayner M (2008) Validating a nutrient profile model. Public Health Nutr 11, 371-378.

34. Committee on Medical Aspects of Food Policy (1991) Dietary Reference Values for Food Energy and Nutrients for the United Kingdom. Report on Health and Social Subjects no. 41. London: Committee on Medical Aspects of Food Policy.

35. Scientific Advisory Committee on Nutrition (2011) Dietary Reference Values for Energy and Nutrients. London: Scientific Advisory Committee on Nutrition. https://www.gov.uk/ government/uploads/system/uploads/attachment_data/file/ 339317/SACN_Dietary_Reference_Values_for_Energy.pdf (accessed November 2014).

36. Scientific Advisory Committee on Nutrition (2015) Carbohydrates and Health Report. London: Scientific Advisory Committee on Nutrition.

37. Elliott P, Vergnaud A-C, Singh D, et al. (2014) The Airwave Health Monitoring Study of police officers and staff in Great Britain: Rationale, design and methods. Environ Res 134C, 280-285.

38. International Physical Activity Questionnaire (2005) International Physical Activity Questionnaire scoring protocol 2005. http://www.ipaq.ki.se/scoring.htm (accessed October 2014).

39. Bingham SA, Gill C, Welch A, et al. (1997) Validation of dietary assessment methods in the UK arm of EPIC using weighed records, and 24-hour urinary nitrogen and potassium and serum vitamin $\mathrm{C}$ and carotenoids as biomarkers. Int $J$ Epidemiol 26, Suppl. 1, S137.

40. Gibson R, Eriksen R, Lamb K, et al. (2017) Dietary assessment of British police force employees: a description of diet record coding procedures and cross-sectional evaluation of dietary energy intake reporting (The Airwave Health Monitoring Study). BMJ Open 7, e012927.

41. Black AE (2000) Critical evaluation of energy intake using the Goldberg cut-off for energy intake: basal metabolic rate. A practical guide to its calculation, use and limitations. Int J Obes Relat Metab Disord 24, 1119-1130.

42. Committee on Medical Aspects of Food Policy (1991) Dietary Reference Values for Food Energy and Nutrients for the United Kingdom. Report on Health and Social Subjects no. 41, pp. 30180. London: Department of Health.

43. Simon A, O'Connell R \& Stephen A (2012) Designing a nutritional scoring system for assessing diet quality for children aged 10 years and under in the UK. Method Innov 7, $27-40$.

44. Rayner M, Scarborough P \& Lobstein T (2009) The UK Ofcom Nutrient Profiling Model. British Heart Foundation Health Promotion Research Group, Department of Public Health, University of Oxford, International Obesity Task Force, London. https://www.ndph.ox.ac.uk/cpnp/files/about/ukofcom-nutrient-profile-model.pdf (accessed December 2015).

45. Scarborough P, Rayner M, Boxer A, et al. (2005) Application of the Nutrient profiling model: Definition of 'fruit, vegetables and nuts' and guidance on quantifying the fruit, vegetable and nut content of a processed product. British Heart Foundation Health Promotion Research Group, Department of Public Health, University of Oxford. https://www.food.gov.uk/sites/ default/files/multimedia/pdfs/nutprofpguide.pdf (accessed October 2014).

46. Rayner M, Scarborough P \& Stockley L (2004) Nutrient Profiles: Options for Definitions for Use in Relation to Food Promotion and Children's Diets. London: Food Standards Agency.

47. Rayner M, Scarborough P \& Williams C (2004) The origin of Guideline Daily Amounts and the Food Standards Agency's guidance on what counts as 'a lot'and 'a little'. Public Health Nutr 7, 549-556.

48. National Collaborating Centre for Chronic Conditions (UK) (2008) Type 2 Diabetes. National Clinical Guideline for Management in Primary and Secondary Care (Update). NICE Clinical Guidelines no. 66. London: Royal College of Physicians.

49. World Health Organization (2000) Obesity: preventing and managing the global epidemic. Report of a WHO consultation. World Health Organ Tech Rep Ser 894, i-253.

50. National Collaborating Centre for Primary Care (2008) Lipid modification: cardiovascular risk assessment and the modification of blood lipids for the primary and secondary prevention of cardiovascular disease. London: National Collaborating Centre for Primary Care.

51. International Expert Committee (2009) Report on the role of the A1C assay in the diagnosis of diabetes. Diabetes Care $\mathbf{3 2}$, $1327-1334$.

52. World Health Organization (2011) Use of glycated haemoglobin (HbA1c) in diagnosis of diabetes mellitus: abbreviated report of a WHO consultation. Geneva: WHO. http://www. who.int/diabetes/publications/report-hba1c_2011.pdf (accessed May 2015).

53. Alcohol Policy Team, Department of Health (2016) How to keep health risks from drinking alcohol to a low level Government response to the public consultation. https://www. gov.uk/government/consultations/health-risks-from-alcoholnew-guidelines (accessed Janaury 2016).

54. World Health Organization (2010) Nutrient Profiling - Report of WHO/IASO Technical Meeting. London: WHO.

55. Sattar N (2008) Why metabolic syndrome criteria have not made prime time: a view from the clinic. Int J Obes 32, S30-S34.

56. Reidlinger DP, Darzi J, Hall WL, et al. (2015) How effective are current dietary guidelines for cardiovascular disease prevention in healthy middle-aged and older men and women? A randomized controlled trial. Am J Clin Nutr 101, 922-930.

57. Julia C, Fézeu LK, Ducrot P, et al. (2015) The nutrient profile of foods consumed using the British Food Standards Agency nutrient profiling system is associated with metabolic syndrome in the SU.VI.MAX Cohort. J Nutr 145, 2355-2361.

58. Nicklas TA, Drewnowski A \& O'Neil CE (2014) The nutrient density approach to healthy eating: challenges and opportunities. Public Health Nutr 17, 2626-2636.

59. Fung TT, Chiuve SE, McCullough ML, et al. (2008) Adherence to a DASH-style diet and risk of coronary heart disease and stroke in women. Arch Intern Med 168, 713-720.

60. Chiuve SE, Sampson L \& Willett WC (2011) Adherence to the Overall Nutritional Quality Index and Risk of Total Chronic Disease. Am J Prev Med 40, 505.

61. Lentjes MA, McTaggart A, Mulligan AA, et al. (2013) Dietary intake measurement using $7 \mathrm{~d}$ diet diaries in British men and women in the European Prospective Investigation into Cancer-Norfolk study: a focus on methodological issues. $\mathrm{Br} \mathrm{J}$ Nutr 111, 516-526.

62. Day NE, McKeown N, Wong M-Y, et al. (2001) Epidemiological assessment of diet: a comparison of a 7-day diary with a food frequency questionnaire using urinary markers of nitrogen, potassium and sodium. Int J Epidemiol 30, 309-317. 
63. Bingham S, Gill C, Welch A, et al. (1994) Comparison of dietary assessment methods in nutritional epidemiology: weighed records v. $24 \mathrm{~h}$ recalls, food-frequency questionnaires and estimated-diet records. $\mathrm{Br} J$ Nutr $\mathbf{7 2}$, 619-643.

64. Health Education Authority (1996) Scientific Basis of Nutrition Education. A Synopsis of Dietary Values. London: Health Education Authority UK.
65. Department of Health, Health and Social Care Information Centre (2013) Health Survey for England - 2012. London: Department of Health, Health and Social Care Information Centre. http://content.digital.nhs.uk/catalogue/PUB13219 (accessed March 2016).

66. Boon N, Boyle R, Bradbury K, et al. (2014) Joint British Societies' consensus recommendations for the prevention of cardiovascular disease (JBS3). Heart 100, Suppl. 2, ii1-ii67. 